\section{PROCEEDINGS A}

royalsocietypublishing.org/journal/rspa

\section{Research}

Cite this article: B Xia, R Zhou. 2020

Time-dependent defects in integrable soliton equations. Proc. R. Soc. A 476: 20190652.

http://dx.doi.org/10.1098/rspa.2019.0652

Received: 10 ctober 2019

Accepted: 13 December 2019

\section{Subject Areas:}

Mathematical physics, applied mathematics

\section{Keywords:}

integrable defect, Bäcklund transformation, soliton equations

\section{Author for correspondence:}

Baoqiang Xia

e-mail: xiabaoqiang@126.com

\section{Time-dependent defects in integrable soliton equations}

\section{Baoqiang Xia and Ruguang Zhou}

School of Mathematics and Statistics, Jiangsu Normal University, Xuzhou, Jiangsu 221116, People's Republic of China

\section{BX, 0000-0003-0522-5164}

We study $(1+1)$-dimensional integrable soliton equations with time-dependent defects located at $x=c(t)$, where $c(t)$ is a function of class $C^{1}$. We define the defect condition as a Bäcklund transformation evaluated at $x=c(t)$ in space rather than over the full line. We show that such a defect condition does not spoil the integrability of the system. We also study soliton solutions that can meet the defect for the system. An interesting discovery is that the defect system admits peaked soliton solutions.

\section{Introduction}

The study of defects, or impurities, in classical $(1+1)$ dimensional integrable field theories has been undertaken intensively in recent years; see, for example, [1-18] and references therein. A defect is usually introduced as a discontinuity at a specific point together with suitable sewing conditions across the defect relating the fields and their derivatives on either side of the defect location. An important question is how to select defect conditions without spoiling the integrability of a system in the presence of defects. A crucial observation was that the defect conditions described by a Bäcklund transformation (BT) frozen at the defect location are compatible with the integrability of a system; this type of defect conditions is able to maintain the conservation of energy and momentum that is expected to guarantee the integrability for the whole system [5-11]. This observation was further exploited to derive the infinite set of conserved quantities and to implement the classical $r$-matrix method to the defect systems [12-17]. The solutions, including soliton and finite-gap solutions, were also derived for the integrable defect systems $[9,18]$. 
We note that the current investigations of the integrable defect problems focused mainly on the case of the defect being at a fixed location; the moving defect problems had received less attention, despite the fact that it was noted in [7] that the defect can move with a constant speed.

The aim of the present paper is to study time-dependent defects in $(1+1)$-dimensional integrable soliton equations, including the nonlinear Schrödinger (NLS) equation, Korteweg-de Vries $(\mathrm{KdV})$ equation and modified $\mathrm{KdV}(\mathrm{mKdV})$ equation belonging to the Ablowitz-KaupNewell-Segur (AKNS) spectral problems [19]. More precisely, we will consider (1+1)-dimensional integrable soliton equations associated with the AKNS system in the presence of a defect at timedependent location $x=c(t)$, where $c(t)$ is a function of class $C^{1}$. Inspired by the case of stationary defects [9], we define the defect condition as a BT fixed at the moving defect location $x=c(t)$ in space rather than over the full line. We establish the Liouville integrability of the resulting defect systems by showing the existence of an infinite set of conserved quantities and by implementing the classical $r$-matrix method. Our results extend the results of $[13,14]$ from the situation of the defect being fixed to the situation of the defect moving with time.

In the present paper, we also study soliton solutions for the time-dependent defect systems. An illustrative example we take is the $\mathrm{KdV}$ equation with an integrable defect that moves with a constant speed. We find that such a defect $\mathrm{KdV}$ equation admits a new type of solutions, the peaked soliton (peakon) solutions, which are continuous in the field variables but with discontinuous derivatives. These solutions to a defect system are reminiscent of peakons discovered in the Camassa-Holm $(\mathrm{CH})$ equation $[20,21]$. We note that peakons for the $\mathrm{CH}$ type equations and peakons presented here should be interpreted in two different senses: the former ones should be interpreted in a suitable weak sense, while the latter ones should be interpreted in the sense that there is a time-dependent defect; see $\S 6$ of the present paper for details.

The paper is organized as follows. In $\$ 2$, we briefly review the construction of the conservation laws and BTs for integrable soliton equations belonging to the AKNS spectral problems. In §3, we present the time-dependent defect systems with defect conditions corresponding to BTs and show such defect systems admit Lagrangian descriptions. In $\S 4$, we study the integrability of the time-dependent defect systems. In $\S 5$, we generalize the results of $\S 4$ to the case that there are multiple time-dependent defects in an integrable system. In $\S 6$, we study soliton solutions for the time-dependent defect $\mathrm{KdV}$ equation. Some concluding remarks are drawn in §7.

\section{AKNS system, conservation laws and BT}

For self-containedness, we start by a brief review of the construction of the conservation laws and the BTs for integrable soliton equations associated with the AKNS spectral problems. We consider the AKNS spectral problems [19]:

$$
\phi_{x}(x, t, \lambda)=U(x, t, \lambda) \phi(x, t, \lambda), \quad U=\left(\begin{array}{cc}
-\mathrm{i} \lambda & u(x, t) \\
v(x, t) & \mathrm{i} \lambda
\end{array}\right)
$$

and

$$
\phi_{t}(x, t, \lambda)=V(x, t, \lambda) \phi(x, t, \lambda), \quad V=\left(\begin{array}{cc}
V_{11} & V_{12} \\
V_{21} & -V_{11}
\end{array}\right),
$$

where $\lambda$ is a spectral parameter, $\phi=\left(\phi_{1}, \phi_{2}\right)^{\mathrm{T}}$ and $V_{j k}, j, k=1,2$, are some functions depending on $u(x, t), v(x, t)$ and on the spectral parameter $\lambda$. For appropriate choices of $V$, the compatibility condition of (2.1), namely the zero curvature condition

$$
U_{t}-V_{x}+[U, V]=0,
$$

may generate quite a few important integrable nonlinear evolution equations. For example,

$$
\begin{array}{ll}
\text { (NLS equation) } & \mathrm{i} u_{t}+u_{x x}-2 \varepsilon u|u|^{2}=0, \quad \varepsilon= \pm 1, \\
\text { (KdV equation) } & u_{t}+u_{x x x}+6 u u_{x}=0
\end{array}
$$

$$
\text { (mKdV equation) } u_{t}+u_{x x x}+6 u^{2} u_{x}=0 \text {, }
$$


are obtained, respectively, by choosing

$$
\left.\begin{array}{l}
v=\varepsilon u^{*}, \quad V_{11}=-2 \mathrm{i} \lambda^{2}-\mathrm{i} \varepsilon|u|^{2}, \quad V_{12}=\varepsilon V_{21}^{*}\left(\lambda^{*}\right)=2 \lambda u+\mathrm{i} u_{x}, \\
v=-1, \quad V_{11}=-4 \mathrm{i} \lambda^{3}+2 \mathrm{i} \lambda u-u_{x}, \quad V_{12}=4 \lambda^{2} u+2 \mathrm{i} \lambda u_{x}-2 u^{2}-u_{x x}, \quad V_{21}=-4 \lambda^{2}+8 u, \\
v=-u, \quad V_{11}=-4 \mathrm{i} \lambda^{3}+2 \mathrm{i} \lambda u^{2}, \quad V_{12}=-V_{21}+4 \mathrm{i} \lambda u_{x}=4 \lambda^{2} u+2 \mathrm{i} \lambda u_{x}-2 u^{3}-u_{x x} .
\end{array}\right\}
$$

This presentation allows one to construct the infinite set of conservation laws associated with the integrable equation. Indeed, denoting $\Gamma=\phi_{2} / \phi_{1}$, we find from (2.1) the following conservation law

$$
(u \Gamma)_{t}=\left(V_{11}+V_{12} \Gamma\right)_{x}
$$

and the following $x$-part and $t$-part Riccati equations

$$
\Gamma_{x}=2 \mathrm{i} \lambda \Gamma+v-u \Gamma^{2}
$$

and

$$
\Gamma_{t}=V_{21}-2 V_{11} \Gamma-V_{12} \Gamma^{2} .
$$

By substituting the expansion $\Gamma=\sum_{n=1}^{\infty} \Gamma_{n}(2 \mathrm{i} \lambda)^{-n}$ into (2.8a) and by equating the coefficients of powers of $\lambda$, we find explicit forms of conservation densities $u \Gamma_{n}$ with

$$
\Gamma_{1}=-v, \quad \Gamma_{2}=-v_{x}, \quad \Gamma_{n+1}=\left(\Gamma_{n}\right)_{x}+u \sum_{j=1}^{n-1} \Gamma_{j} \Gamma_{n-j}, \quad n \geq 2 .
$$

We now consider BTs for the AKNS system. We introduce another copy of the auxiliary problems for $\tilde{\phi}$ with Lax pair $\tilde{U}, \tilde{V}$ defined as in (2.1) with the new potentials $\tilde{u}, \tilde{v}$, replacing $u, v$. We assume that the two systems are related by a Darboux transformation (DT) [22],

$$
\tilde{\phi}(x, t, \lambda)=B(x, t, \lambda) \phi(x, t, \lambda),
$$

where the matrix $B(x, t, \lambda)$ satisfies

$$
B_{x}(x, t, \lambda)=\tilde{U}(x, t, \lambda) B(x, t, \lambda)-B(x, t, \lambda) U(x, t, \lambda)
$$

and

$$
B_{t}(x, t, \lambda)=\tilde{V}(x, t, \lambda) B(x, t, \lambda)-B(x, t, \lambda) V(x, t, \lambda) .
$$

Equation (2.11) induces a relation, called a BT [23], between the potentials $u, v$ and $\tilde{u}, \tilde{v}$. For example, for the NLS equation (2.3), we may take

$$
B=I+\frac{1}{2 \lambda}\left(\begin{array}{cc}
\alpha+\mathrm{i} \Omega & -\mathrm{i}(\tilde{u}-u) \\
\mathrm{i} \varepsilon(\tilde{u}-u)^{*} & \alpha-\mathrm{i} \Omega
\end{array}\right), \quad \Omega= \pm \sqrt{\beta^{2}+\varepsilon|\tilde{u}-u|^{2}},
$$

the corresponding BT becomes

and

$$
\left.\begin{array}{l}
\tilde{u}_{x}-u_{x}=i \alpha(\tilde{u}-u)+\Omega(\tilde{u}+u) \\
\tilde{u}_{t}-u_{t}=-\alpha\left(\tilde{u}_{x}-u_{x}\right)+i \Omega\left(\tilde{u}_{x}+u_{x}\right)-\mathrm{i} \varepsilon(\tilde{u}-u)\left(|\tilde{u}|^{2}+|u|^{2}\right),
\end{array}\right\}
$$

where $\alpha$ and $\beta$ are two arbitrary real constants. For the KdV equation (2.4), we may take

$$
B=I+\mathrm{i} \lambda^{-1}\left(\begin{array}{cc}
\frac{1}{2} \sqrt{\beta^{2}-2(\tilde{u}+u)} & \frac{1}{2}(\tilde{u}+u) \\
1 & -\frac{1}{2} \sqrt{\beta^{2}-2(\tilde{u}+u)}
\end{array}\right),
$$

the corresponding BT becomes

and

$$
\left.\begin{array}{l}
\left(\tilde{u}_{x}+u_{x}\right)=(\tilde{u}-u) \sqrt{\beta^{2}-2(\tilde{u}+u)} \\
\left(\tilde{u}_{t}+u_{t}\right)=-\left(3\left(\tilde{u}^{2}-u^{2}\right)+(\tilde{u}-u)_{x x}\right) \sqrt{\beta^{2}-2(\tilde{u}+u)} .
\end{array}\right\}
$$


For the mKdV equation (2.5), we may take

$$
B=I+\frac{\mathrm{i}}{2} \lambda^{-1}\left(\begin{array}{cc}
\sqrt{\beta^{2}-(\tilde{u}-u)^{2}} & -(\tilde{u}-u) \\
-(\tilde{u}-u) & -\sqrt{\beta^{2}-(\tilde{u}-u)^{2}}
\end{array}\right),
$$

the corresponding BT becomes

and

$$
\left.\begin{array}{l}
\left(\tilde{u}_{x}-u_{x}\right)=(\tilde{u}+u) \sqrt{\beta^{2}-(\tilde{u}-u)^{2}} \\
\left(\tilde{u}_{t}-u_{t}\right)=-\left(2\left(\tilde{u}^{3}+u^{3}\right)+(\tilde{u}+u)_{x x}\right) \sqrt{\beta^{2}-(\tilde{u}-u)^{2}} .
\end{array}\right\}
$$

\section{Integrable systems with time-dependent defects}

Let $c(t)$ be a function of class $C^{1}$. We study integrable equations with a time-dependent defect placing at $x=c(t)$ in space. Following the idea in the case of stationary defects, we define the defect condition as a BT evaluated at the moving location $x=c(t)$. We show such a defect system admits a Lagrangian description.

\section{(a) Time-dependent defect conditions arising from BTs}

We suppose that the auxiliary problem (2.1) exists for $x>c(t)$, while the one for $\tilde{U}$ and $\tilde{V}$ exists for $x<c(t)$. At the time-dependent position $x=c(t)$, we assume that the two systems are connected via the condition (2.10) evaluated at $x=c(t)$.

Definition 3.1. A $(1+1)$-dimensional integrable equation with a defect at time-dependent location $x=c(t)$ in space is described by the following internal boundary problem:

$-u(x, t)$ and $\tilde{u}(x, t)$ satisfy the equation in the bulk for $x>c(t)$ and for $x<c(t)$, respectively;

- at $x=c(t), u(c(t), t)$ and $\tilde{u}(c(t), t)$ are connected by a condition corresponding to the BT for $u(x, t)$ and $\tilde{u}(x, t)$.

For example, using the BTs introduced in §2, the NLS equation with the above-defined timedependent defect reads

$$
\begin{aligned}
& \mathrm{i} u_{t}+u_{x x}-2 \varepsilon u|u|^{2}=0, \quad \varepsilon= \pm 1, x>c(t), \\
& \mathrm{i} \tilde{u}_{t}+\tilde{u}_{x x}-2 \varepsilon \tilde{u}|\tilde{u}|^{2}=0, \quad \varepsilon= \pm 1, \quad x<c(t), \\
& \left.\left(\tilde{u}_{x}-u_{x}\right)\right|_{x=c(t)}=\left.(\mathrm{i} \alpha(\tilde{u}-u)+\Omega(\tilde{u}+u))\right|_{x=c(t)}
\end{aligned}
$$

and

$$
\left.\left(\tilde{u}_{t}-u_{t}\right)\right|_{x=c(t)}=\left.\left(-\alpha\left(\tilde{u}_{x}-u_{x}\right)+\mathrm{i} \Omega\left(\tilde{u}_{x}+u_{x}\right)-\mathrm{i} \varepsilon(\tilde{u}-u)\left(|\tilde{u}|^{2}+|u|^{2}\right)\right)\right|_{x=c(t)},
$$

where $\Omega= \pm \sqrt{\beta^{2}+\varepsilon|\tilde{u}-u|^{2}}$. The $\mathrm{KdV}$ equation with the time-dependent defect reads

$$
\begin{aligned}
& u_{t}+u_{x x x}+6 u u_{x}=0, \quad x>c(t) \\
& \tilde{u}_{t}+\tilde{u}_{x x x}+6 \tilde{u} \tilde{u}_{x}=0, \quad x<c(t) \\
& \left.\left(\tilde{u}_{x}+u_{x}\right)\right|_{x=c(t)}=\left.(\tilde{u}-u) \sqrt{\beta^{2}-2(\tilde{u}+u)}\right|_{x=c(t)}
\end{aligned}
$$

and

$$
\left.\left(\tilde{u}_{t}+u_{t}\right)\right|_{x=c(t)}=-\left.\left(3\left(\tilde{u}^{2}-u^{2}\right)+(\tilde{u}-u)_{x x}\right) \sqrt{\beta^{2}-2(\tilde{u}+u)}\right|_{x=c(t)} .
$$


The $\mathrm{mKdV}$ equation with the time-dependent defect reads

$$
\begin{aligned}
& u_{t}+u_{x x x}+6 u^{2} u_{x}=0, \quad x>c(t), \\
& \tilde{u}_{t}+\tilde{u}_{x x x}+6 \tilde{u}^{2} \tilde{u}_{x}=0, \quad x<c(t), \\
& \left.\left(\tilde{u}_{x}-u_{x}\right)\right|_{x=c(t)}=\left.(\tilde{u}+u) \sqrt{\beta^{2}-(\tilde{u}-u)^{2}}\right|_{x=c(t)}
\end{aligned}
$$

and

$$
\left.\left(\tilde{u}_{t}-u_{t}\right)\right|_{x=c(t)}=-\left.\left(2\left(\tilde{u}^{3}+u^{3}\right)+(\tilde{u}+u)_{x x}\right) \sqrt{\beta^{2}-(\tilde{u}-u)^{2}}\right|_{x=c(t)} .
$$

\section{(b) Lagrangian descriptions for the defect systems}

We now show that the time-dependent defect system also admits a Lagrangian description. We will fix our ideas on the above-mentioned three examples: the defect NLS equation (3.1), the defect $\mathrm{KdV}$ equation (3.2) and the defect $\mathrm{mKdV}$ equation (3.3).

\section{(i) Lagrangian formulation for the defect NLS equation}

The NLS equation (2.3) in the bulk is described by the Lagrangian

$$
L=\int_{-\infty}^{\infty} \mathrm{d} x\left(\frac{\mathrm{i}}{2}\left(u^{*} u_{t}-u u_{t}^{*}\right)-\left|u_{x}\right|^{2}-\varepsilon|u|^{4}\right)
$$

To describe the defect NLS equation (3.1), we modify the Lagrangian as follows

$$
L=\int_{-\infty}^{c(t)} \mathrm{d} x \mathcal{L}(\tilde{u})+D+\int_{\mathcal{c}(t)}^{\infty} \mathrm{d} x \mathcal{L}(u),
$$

where

$$
\mathcal{L}(u)=\frac{\mathrm{i}}{2}\left(u^{*} u_{t}-u u_{t}^{*}\right)-\left|u_{x}\right|^{2}-\varepsilon|u|^{4}
$$

is the Lagrangian density of the bulk system for $x>c(t)$,

$$
\mathcal{L}(\tilde{u})=\frac{\mathrm{i}}{2}\left(\tilde{u}^{*} \tilde{u}_{t}-\tilde{u} \tilde{u}_{t}^{*}\right)-\left|\tilde{u}_{x}\right|^{2}-\varepsilon|\tilde{u}|^{4}
$$

is the Lagrangian density of the bulk system for $x<c(t)$,

$$
\begin{aligned}
D= & -\frac{\mathrm{i}}{2} \varepsilon \omega\left(\frac{\dot{\tilde{\mathfrak{u}}}-\dot{\mathfrak{u}}}{\tilde{\mathfrak{u}}-\mathfrak{u}}-\frac{\dot{\tilde{\mathfrak{u}}}^{*}-\dot{\mathfrak{u}}^{*}}{\tilde{\mathfrak{u}}^{*}-\mathfrak{u}^{*}}\right)-\frac{1}{3} \varepsilon \omega^{3}+\omega\left(|\tilde{\mathfrak{u}}|^{2}+|\mathfrak{u}|^{2}+\varepsilon \alpha^{2}-\varepsilon \alpha c^{\prime}(t)\right) \\
& +\left(\frac{\dot{\mathrm{i}}}{2} c^{\prime}(t)-\mathrm{i} \alpha\right)\left(\tilde{\mathfrak{u}}^{*} \mathfrak{u}-\tilde{\mathfrak{u}} \mathfrak{u}^{*}\right)
\end{aligned}
$$

is the defect contribution at $x=c(t)$. In (3.8), we used the following abbreviated expressions

and

$$
\begin{aligned}
& \mathfrak{u}=u(c(t), t), \quad \tilde{\mathfrak{u}}=\tilde{u}(c(t), t), \quad \omega= \pm \sqrt{\beta^{2}+\varepsilon|\tilde{\mathfrak{u}}-\mathfrak{u}|^{2}}, \\
& \mathfrak{u}_{1}=\left.u_{x}(x, t)\right|_{x=c(t)}, \quad \tilde{\mathfrak{u}}_{1}=\left.\tilde{u}_{x}(x, t)\right|_{x=c(t)}, \\
& \mathfrak{u}_{2}=\left.u_{t}(x, t)\right|_{x=c(t)}, \quad \tilde{\mathfrak{u}}_{2}=\left.\tilde{u}_{t}(x, t)\right|_{x=c(t)} \\
& \dot{\mathfrak{u}}=\frac{\mathrm{d} u(c(t), t)}{\mathrm{d} t}=c^{\prime}(t) \mathfrak{u}_{1}+\mathfrak{u}_{2}, \quad \dot{\tilde{\mathfrak{u}}}=\frac{\mathrm{d} \tilde{u}(c(t), t)}{\mathrm{d} t}=c^{\prime}(t) \tilde{\mathfrak{u}}_{1}+\tilde{\mathfrak{u}}_{2} .
\end{aligned}
$$

Claim 3.2. The defect NLS equation (3.1) can be described by the Lagrangian (3.5). 
Indeed, we consider the complete action

$$
\mathcal{A}=\int_{-\infty}^{\infty} \mathrm{d} t\left\{\int_{-\infty}^{c(t)} \mathrm{d} x \mathcal{L}(\tilde{u})+D+\int_{c(t)}^{\infty} \mathrm{d} x \mathcal{L}(u)\right\}
$$

The variation of $\mathcal{A}$ with respect to $u^{*}$ gives

$$
\delta \mathcal{A}=\int_{-\infty}^{\infty} \mathrm{d} t\left\{\int_{c(t)}^{\infty} \mathrm{d} x\left(\frac{\partial \mathcal{L}(u)}{\partial u^{*}} \delta u^{*}+\frac{\partial \mathcal{L}(u)}{\partial u_{x}^{*}} \delta u_{x}^{*}+\frac{\partial \mathcal{L}(u)}{\partial u_{t}^{*}} \delta u_{t}^{*}\right)+\frac{\partial D}{\partial \mathfrak{u}^{*}} \delta \mathfrak{u}^{*}+\frac{\partial D}{\partial \dot{\mathfrak{u}}^{*}} \delta \dot{\mathfrak{u}}^{*}\right\}
$$

Integrating the second term in (3.11) by parts with respect to $x$, we find

$$
\begin{aligned}
& \int_{-\infty}^{\infty} \mathrm{d} t \int_{\mathcal{c}(t)}^{\infty} \mathrm{d} x\left(\frac{\partial \mathcal{L}(u)}{\partial u_{x}^{*}} \delta u_{x}^{*}\right) \\
& =-\int_{-\infty}^{\infty} \mathrm{d} t\left\{\left.\left(\frac{\partial \mathcal{L}(u)}{\partial u_{x}^{*}} \delta u^{*}\right)\right|_{x=c(t)}+\int_{\mathcal{c}(t)}^{\infty} \mathrm{d} x\left(\left(\frac{\partial \mathcal{L}(u)}{\partial u_{x}^{*}}\right)_{x} \delta u^{*}\right)\right\} \\
& =\int_{-\infty}^{\infty} \mathrm{d} t\left\{\mathfrak{u}_{1} \delta u^{*}-\int_{c(t)}^{\infty} \mathrm{d} x\left(\left(\frac{\partial \mathcal{L}(u)}{\partial u_{x}^{*}}\right)_{x} \delta u^{*}\right)\right\}
\end{aligned}
$$

Using the identity

$$
\begin{aligned}
& \frac{\mathrm{d}}{\mathrm{d} t}\left(\int_{c(t)}^{\infty} \mathrm{d} x\left(\frac{\partial \mathcal{L}(u)}{\partial u_{t}^{*}} \delta u^{*}\right)\right) \\
& \quad=-\left.c^{\prime}(t) \frac{\partial \mathcal{L}(u)}{\partial u_{t}^{*}} \delta u^{*}\right|_{x=c(t)}+\int_{\mathcal{c}(t)}^{\infty} \mathrm{d} x\left(\left(\frac{\partial \mathcal{L}(u)}{\partial u_{t}^{*}}\right)_{t} \delta u^{*}+\frac{\partial \mathcal{L}(u)}{\partial u_{t}^{*}} \delta u_{t}^{*}\right) \\
& \quad=\frac{\mathrm{i}}{2} c^{\prime}(t) \mathfrak{u} \delta \mathfrak{u}^{*}+\int_{c(t)}^{\infty} \mathrm{d} x\left(\left(\frac{\partial \mathcal{L}(u)}{\partial u_{t}^{*}}\right)_{t} \delta u^{*}+\frac{\partial \mathcal{L}(u)}{\partial u_{t}^{*}} \delta u_{t}^{*}\right)
\end{aligned}
$$

the third term in (3.11) can be written as

$$
\int_{-\infty}^{\infty} \mathrm{d} t \int_{c(t)}^{\infty} \mathrm{d} x\left(\frac{\partial \mathcal{L}(u)}{\partial u_{t}^{*}} \delta u_{t}^{*}\right)=-\int_{-\infty}^{\infty} \mathrm{d} t\left\{\frac{\mathrm{i}}{2} c^{\prime}(t) \mathfrak{u} \delta \mathfrak{u}^{*}+\int_{c(t)}^{\infty} \mathrm{d} x\left(\left(\frac{\partial \mathcal{L}(u)}{\partial u_{t}^{*}}\right)_{t} \delta u^{*}\right)\right\} .
$$

Integrating the last term in (3.11) by parts with respect to $t$, we have

$$
\int_{-\infty}^{\infty} \mathrm{d} t\left(\frac{\partial D}{\partial \dot{\mathfrak{u}}^{*}} \delta \dot{\mathfrak{u}}^{*}\right)=-\int_{-\infty}^{\infty} \mathrm{d} t\left(\delta \mathfrak{u}^{*} \frac{\mathrm{d}}{\mathrm{d} t}\left(\frac{\partial D}{\partial \dot{\mathfrak{u}}^{*}}\right)\right)
$$

Inserting (3.12), (3.14) and (3.15) into (3.11) and requiring the variation to be stationary, we obtain

$$
\begin{aligned}
0= & \int_{-\infty}^{\infty} \mathrm{d} t \int_{\mathcal{C}(t)}^{\infty} \mathrm{d} x\left[\delta u^{*}\left(\frac{\partial \mathcal{L}(u)}{\partial u^{*}}-\frac{\partial}{\partial x}\left(\frac{\partial \mathcal{L}(u)}{\partial u_{x}^{*}}\right)-\frac{\partial}{\partial t}\left(\frac{\partial \mathcal{L}(u)}{\partial u_{t}^{*}}\right)\right)\right] \\
& +\int_{-\infty}^{\infty} \mathrm{d} t\left[\delta u^{*}\left(\mathfrak{u}_{1}-\frac{\mathrm{i}}{2} c^{\prime}(t) \mathfrak{u}+\frac{\partial D}{\partial \mathfrak{u}^{*}}-\frac{\mathrm{d}}{\mathrm{d} t}\left(\frac{\partial D}{\partial \dot{\mathfrak{u}}^{*}}\right)\right)\right] .
\end{aligned}
$$

Similarly, requiring the variation of (3.10) with respect to $\tilde{u}^{*}$ to be stationary gives

$$
\begin{aligned}
0= & \int_{-\infty}^{\infty} \mathrm{d} t \int_{-\infty}^{c(t)} \mathrm{d} x\left[\delta \tilde{u}^{*}\left(\frac{\partial \mathcal{L}(\tilde{u})}{\partial \tilde{u}^{*}}-\frac{\partial}{\partial x}\left(\frac{\partial \mathcal{L}(\tilde{u})}{\partial \tilde{u}_{x}^{*}}\right)-\frac{\partial}{\partial t}\left(\frac{\partial \mathcal{L}(\tilde{u})}{\partial \tilde{u}_{t}^{*}}\right)\right)\right] \\
& +\int_{-\infty}^{\infty} \mathrm{d} t\left[\delta \tilde{u}^{*}\left(-\tilde{\mathfrak{u}}_{1}+\frac{\mathrm{i}}{2} c^{\prime}(t) \tilde{\mathfrak{u}}+\frac{\partial D}{\partial \tilde{\mathfrak{u}}^{*}}-\frac{\mathrm{d}}{\mathrm{d} t}\left(\frac{\partial D}{\partial \dot{\tilde{u}}^{*}}\right)\right)\right] .
\end{aligned}
$$




$$
\begin{aligned}
& \frac{\partial \mathcal{L}(u)}{\partial u^{*}}-\frac{\partial}{\partial x}\left(\frac{\partial \mathcal{L}(u)}{\partial u_{x}^{*}}\right)-\frac{\partial}{\partial t}\left(\frac{\partial \mathcal{L}(u)}{\partial u_{t}^{*}}\right)=0, \quad x>c(t), \\
& \frac{\partial \mathcal{L}(\tilde{u})}{\partial \tilde{u}^{*}}-\frac{\partial}{\partial x}\left(\frac{\partial \mathcal{L}(\tilde{u})}{\partial \tilde{u}_{x}^{*}}\right)-\frac{\partial}{\partial t}\left(\frac{\partial \mathcal{L}(\tilde{u})}{\partial \tilde{u}_{t}^{*}}\right)=0, \quad x<c(t), \\
& \mathfrak{u}_{1}-\frac{\mathrm{i}}{2} c^{\prime}(t) \mathfrak{u}+\frac{\partial D}{\partial \mathfrak{u}^{*}}-\frac{\mathrm{d}}{\mathrm{d} t}\left(\frac{\partial D}{\partial \dot{\mathfrak{u}}^{*}}\right)=0 \\
& \tilde{\mathfrak{u}}_{1}-\frac{\mathrm{i}}{2} c^{\prime}(t) \tilde{\mathfrak{u}}-\frac{\partial D}{\partial \tilde{\mathfrak{u}}^{*}}+\frac{\mathrm{d}}{\mathrm{d} t}\left(\frac{\partial D}{\partial \dot{\tilde{\mathfrak{u}}}^{*}}\right)=0 .
\end{aligned}
$$

Equations (3.18a) and (3.18b) give nothing but (3.1a) and (3.1b), while (3.18c) and (3.18d), after some algebra, give exactly the defect conditions (3.1c) and (3.1d) at the defect location.

\section{(ii) Lagrangian formulation for the defect KdV equation}

For the $\mathrm{KdV}$ equation, the setting $u=q_{x}$ is suitable for a Lagrangian description. In this setting, the defect $\mathrm{KdV}$ equation (3.2) can be rewritten as

$$
\begin{aligned}
& q_{t}+q_{x x x}+3 q_{x}^{2}=0, \quad x>c(t), \\
& \tilde{q}_{t}+\tilde{q}_{x x x}+3 \tilde{q}_{x}^{2}=0, \quad x<c(t), \\
& \left.\left(\tilde{q}_{x}+q_{x}\right)\right|_{x=c(t)}=-2 \alpha-\left.\frac{1}{2}(\tilde{q}-q)^{2}\right|_{x=c(t)}
\end{aligned}
$$

and

$$
\left.\left(\tilde{q}_{t}+q_{t}\right)\right|_{x=c(t)}=\left.\left[\left(\tilde{q}_{x x}-q_{x x}\right)(\tilde{q}-q)-2\left(\left(\tilde{q}_{x}\right)^{2}+\left(q_{x}\right)^{2}+\tilde{q}_{x} q_{x}\right)\right]\right|_{x=c(t)} .
$$

where $\alpha=-\beta^{2} / 4$. Regarding this defect system, we introduce the Lagrangian

$$
L=\int_{-\infty}^{c(t)} \mathrm{d} x \mathcal{L}(\tilde{q})+D+\int_{c(t)}^{\infty} \mathrm{d} x \mathcal{L}(q)
$$

where

$$
\mathcal{L}(q)=\frac{1}{2} q_{x} q_{t}+\left(q_{x}\right)^{3}-\frac{1}{2}\left(q_{x x}\right)^{2}
$$

is the Lagrangian density of the bulk system for $x>c(t)$,

$$
\mathcal{L}(\tilde{q})=\frac{1}{2} \tilde{q}_{x} \tilde{q}_{t}+\left(\tilde{q}_{x}\right)^{3}-\frac{1}{2}\left(\tilde{q}_{x x}\right)^{2}
$$

is the Lagrangian density of the bulk system for $x<c(t)$,

$$
\begin{aligned}
D= & \frac{1}{4}(\dot{\mathfrak{q}} \dot{\tilde{\mathfrak{q}}}-\tilde{\mathfrak{q}} \dot{\mathfrak{q}})-\frac{9}{40}(\tilde{\mathfrak{q}}-\mathfrak{q})^{5}-(\tilde{\mathfrak{q}}-\mathfrak{q})^{3}\left(2 \alpha+\frac{3}{4}\left(\tilde{\mathfrak{q}}_{1}+\mathfrak{q}_{1}\right)\right)+\frac{1}{4}(\tilde{\mathfrak{q}}-\mathfrak{q})^{2}\left(\tilde{\mathfrak{q}}_{11}-\mathfrak{q}_{11}\right) \\
& -(\tilde{\mathfrak{q}}-\mathfrak{q})\left(\left(\tilde{\mathfrak{q}}_{1}\right)^{2}+\left(\mathfrak{q}_{1}\right)^{2}+\tilde{\mathfrak{q}}_{1} \mathfrak{q}_{1}+3 \alpha\left(\tilde{\mathfrak{q}}_{1}+\mathfrak{q}_{1}\right)+6 \alpha^{2}\right)+\frac{1}{2}\left(\tilde{\mathfrak{q}}_{11}-\mathfrak{q}_{11}\right)\left(\tilde{\mathfrak{q}}_{1}+\mathfrak{q}_{1}+2 \alpha\right) \\
& -c^{\prime}(t)(\tilde{\mathfrak{q}}-\mathfrak{q})\left(\alpha+\frac{1}{12}(\tilde{\mathfrak{q}}-\mathfrak{q})^{2}\right)
\end{aligned}
$$

is the defect contribution at $x=c(t)$. In (3.23), we have used the following abbreviated expressions:

and

$$
\begin{aligned}
& \mathfrak{q}=q(c(t), t), \quad \tilde{\mathfrak{q}}=\tilde{q}(c(t), t), \\
& \mathfrak{q}_{1}=\left.q_{x}(x, t)\right|_{x=c(t)}, \quad \tilde{\mathfrak{q}}_{1}=\left.\tilde{q}_{x}(x, t)\right|_{x=c(t)}, \\
& \mathfrak{q}_{11}=\left.q_{x x}(x, t)\right|_{x=c(t)}, \quad \tilde{\mathfrak{q}}_{11}=\left.\tilde{q}_{x x}(x, t)\right|_{x=c(t)}, \\
& \mathfrak{q}_{2}=\left.q_{t}(x, t)\right|_{x=c(t)}, \quad \tilde{\mathfrak{q}}_{2}=\left.\tilde{q}_{t}(x, t)\right|_{x=c(t)}
\end{aligned}
$$


In analogy to the case of the NLS equation, by requiring the variation of the complete action $\int_{-\infty}^{\infty} \mathrm{d} t L$ to be stationary with respect to $q$ or $\tilde{q}$, we find the following defect conditions:

and

$$
\frac{1}{2} \dot{\mathfrak{q}}=-\frac{\partial D}{\partial \mathfrak{q}}+\frac{\mathrm{d}}{\mathrm{d} t}\left(\frac{\partial D}{\partial \dot{\mathfrak{q}}}\right), \quad \mathfrak{q}_{11}+\frac{\partial D}{\partial \mathfrak{q}_{1}}=0, \quad \frac{\partial D}{\partial \mathfrak{q}_{11}}=0,
$$

$$
\frac{1}{2} \dot{\tilde{\mathfrak{q}}}=\frac{\partial D}{\partial \tilde{\mathfrak{q}}}-\frac{\mathrm{d}}{\mathrm{d} t}\left(\frac{\partial D}{\partial \dot{\tilde{\mathfrak{q}}}}\right), \quad-\tilde{\mathfrak{q}}_{11}+\frac{\partial D}{\partial \tilde{\mathfrak{q}}_{1}}=0, \quad \frac{\partial D}{\partial \tilde{\mathfrak{q}}_{11}}=0
$$

Using (3.23), the above defect conditions are exactly equivalent to (3.19c) and (3.19d). To sum up, we find

Claim 3.3. The defect KdV equation (3.19) can be described by the Lagrangian (3.20).

\section{(iii) Lagrangian formulation for the defect mKdV equation}

For the $\mathrm{mKdV}$ equation in the setting $u=q_{x}$, an alternative Darboux matrix $B$ can be taken as

$$
B=I+\frac{\mathrm{i} \beta}{2 \lambda}\left(\begin{array}{cc}
\cos (\tilde{q}-q) & -\sin (\tilde{q}-q) \\
-\sin (\tilde{q}-q) & -\cos (\tilde{q}-q)
\end{array}\right),
$$

and the corresponding BT becomes [9]

and

$$
\left.\begin{array}{l}
\left(\tilde{q}_{x}+q_{x}\right)=\beta \sin (\tilde{q}-q) \\
\left(\tilde{q}_{t}+q_{t}\right)=-\beta\left[\left(\tilde{q}_{x x}-q_{x x}\right) \cos (\tilde{q}-q)+\left(\tilde{q}_{x}^{2}+q_{x}^{2}\right) \sin (\tilde{q}-q)\right] .
\end{array}\right\}
$$

Then the time-dependent defect $\mathrm{mKdV}$ equation in the potential $q$ reads

$$
\begin{aligned}
& q_{t}+q_{x x x}+2\left(q_{x}\right)^{3}=0, \quad x>c(t), \\
& q_{t}+q_{x x x}+2\left(q_{x}\right)^{3}=0, \quad x<c(t), \\
& \left.\left(\tilde{q}_{x}+q_{x}\right)\right|_{x=c(t)}=\left.\beta \sin (\tilde{q}-q)\right|_{x=c(t)}
\end{aligned}
$$

and

$$
\left.\left(\tilde{q}_{t}+q_{t}\right)\right|_{x=c(t)}=-\left.\beta\left[\left(\tilde{q}_{x x}-q_{x x}\right) \cos (\tilde{q}-q)+\left(\tilde{q}_{x}^{2}+q_{x}^{2}\right) \sin (\tilde{q}-q)\right]\right|_{x=c(t)} .
$$

We introduce the Lagrangian

$$
L=\int_{-\infty}^{c(t)} \mathrm{d} x \mathcal{L}(\tilde{q})+D+\int_{\mathcal{c}(t)}^{\infty} \mathrm{d} x \mathcal{L}(q)
$$

where

$$
\mathcal{L}(q)=\frac{1}{2} q_{x} q_{t}+\frac{1}{2}\left(q_{x}\right)^{4}-\frac{1}{2}\left(q_{x x}\right)^{2}
$$

is the Lagrangian density of the bulk system for $x>c(t)$,

$$
\mathcal{L}(\tilde{q})=\frac{1}{2} \tilde{q}_{x} \tilde{q}_{t}+\frac{1}{2}\left(\tilde{q}_{x}\right)^{4}-\frac{1}{2}\left(\tilde{q}_{x x}\right)^{2}
$$

is the Lagrangian density of the bulk system for $x<c(t)$,

$$
\begin{aligned}
D= & \frac{1}{4}(\mathfrak{q} \dot{\tilde{\mathfrak{q}}}-\tilde{\mathfrak{q}} \dot{\mathfrak{q}})+\frac{1}{2}\left(\tilde{\mathfrak{q}}_{11}-\mathfrak{q}_{11}\right)\left(\tilde{\mathfrak{q}}_{1}+\mathfrak{q}_{1}-\beta \sin (\tilde{\mathfrak{q}}-\mathfrak{q})\right)-\frac{1}{2} \beta c^{\prime}(t) \cos (\tilde{\mathfrak{q}}-\mathfrak{q}) \\
& +\frac{\beta}{6} \cos (\tilde{\mathfrak{q}}-\mathfrak{q})\left[\left(\tilde{\mathfrak{q}}_{1}\right)^{2}+\left(\mathfrak{q}_{1}\right)^{2}-4 \tilde{\mathfrak{q}}_{1} \mathfrak{q}_{1}+\beta\left(\tilde{\mathfrak{q}}_{1}+\mathfrak{q}_{1}\right) \sin (\tilde{\mathfrak{q}}-\mathfrak{q})+\beta^{2}\right]
\end{aligned}
$$

is the defect contribution at $x=c(t)$. In (3.32), we have used the same abbreviated expressions as used in the case of defect $\mathrm{KdV}$ equation (3.24). In analogy to the case of defect $\mathrm{KdV}$ equation, we find

Claim 3.4. The defect $m K d V$ equation (3.28) can be described by the Lagrangian (3.29).

Remark 3.5. Taking $c^{\prime}(t)=0$ in (3.5), (3.20) and (3.29) respectively, from our Lagrangian formulations for the time-dependent defect systems we can recover the corresponding Lagrangian formulations for the stationary defect systems [9]. 


\section{Integrability of the time-dependent defect system}

In this section, we will establish the integrability of the defect system both by constructing an infinite set of conserved densities and by implementing the classical $r$-matrix method. This analysis is based on an extension of the results in [13,14] from the situation of the stationary defect to the situation of the moving defect.

\section{(a) Conservation laws}

By generalizing the analogous result of [13], we find the following conservation densities for the time-dependent defect system.

Proposition 4.1. The generating function for the integrals of the motion reads

$$
I(\lambda)=I_{\text {bulk }}^{\text {left }}(\lambda)+I_{\text {bulk }}^{\text {right }}(\lambda)+I_{\text {defect }}(\lambda),
$$

where

and

$$
\begin{array}{r}
I_{\text {bulk }}^{\text {left }}(\lambda)=\int_{-\infty}^{c(t)} \tilde{u} \tilde{\Gamma} \mathrm{d} x, \\
I_{\text {bulk }}^{\text {right }}(\lambda)=\int_{c(t)}^{\infty} u \Gamma \mathrm{d} x
\end{array}
$$

and $B_{j k}, j, k=1,2$, is the jk-entry of the defect matrix $B$.

Proof. From (2.7), we have

$$
(u \Gamma)_{t}=\left(V_{11}+V_{12} \Gamma\right)_{x}, \quad x>c(t)
$$

and

$$
(\tilde{u} \tilde{\Gamma})_{t}=\left(\tilde{V}_{11}+\tilde{V}_{12} \tilde{\Gamma}\right)_{x}, \quad x<c(t),
$$

where $\tilde{\Gamma}=\tilde{\phi}_{2} / \tilde{\phi}_{1}$. Using (4.5) and the rapid decay of the fields $u(x, t), v(x, t), \tilde{u}(x, t), \tilde{v}(x, t)$, we find

$$
\left(\int_{-\infty}^{c(t)} \tilde{u} \tilde{\Gamma} \mathrm{d} x+\int_{c(t)}^{\infty} u \Gamma \mathrm{d} x\right)_{t}=\left.\left(\tilde{V}_{11}+\tilde{V}_{12} \tilde{\Gamma}-V_{11}-V_{12} \Gamma\right)\right|_{x=c(t)}+\left.c^{\prime}(t)(\tilde{u} \tilde{\Gamma}-u \Gamma)\right|_{x=c(t)} .
$$

From (2.10), we have

$$
\left.\tilde{\Gamma}\right|_{x=c(t)}=\left.\frac{B_{21}+B_{22} \Gamma}{B_{11}+B_{12} \Gamma}\right|_{x=c(t)} .
$$

Using (2.8b), (2.11b) and (4.7), we obtain

$$
\left.\left(\tilde{V}_{11}+\tilde{V}_{12} \tilde{\Gamma}-V_{11}-V_{12} \Gamma\right)\right|_{x=c(t)}=\left.\frac{\left(B_{11}+B_{12} \Gamma\right)_{t}}{B_{11}+B_{12} \Gamma}\right|_{x=c(t)} .
$$

Using (2.8a), (2.11a) and (4.7), we obtain

$$
\left.(\tilde{u} \tilde{\Gamma}-u \Gamma)\right|_{x=c(t)}=\left.\frac{\left(B_{11}+B_{12} \Gamma\right)_{x}}{B_{11}+B_{12} \Gamma}\right|_{x=c(t)} .
$$

Substituting (4.8) and (4.9) into (4.6), we obtain

$$
\left(\int_{-\infty}^{c(t)} \tilde{u} \tilde{\Gamma} \mathrm{d} x+\int_{c(t)}^{\infty} u \Gamma \mathrm{d} x\right)_{t}=\left(\left.\ln \left(B_{11}+B_{12} \Gamma\right)\right|_{x=c(t)}\right)_{t}
$$

Thus,

$$
(I(\lambda))_{t}=0 .
$$

This completes the proof. 
Remark 4.2. Proposition 4.1 implies that the integrals of motion for the time-dependent defect system take a very similar form as those for the stationary defect system [13]. However, the proof for proposition 4.1 is technically more involved than analogous proof for the case of the stationary defect (see $\S 1.2$ in [13]); we need to pay more attention to the $t$-derivatives of the associated quantities.

\section{(b) Canonical transformation and classical $r$-matrix approach}

Canonical properties of BTs to integrable nonlinear evolution equations have been established in $[24,25]$. Recently, by introducing a new Poisson bracket (called equal-space bracket), it was shown in [14] that a defect condition described by a frozen BT can be interpreted naturally as a canonical transformation of the system. As a consequence, the classical $r$-matrix approach [26-28] can be implemented to establish Liouville integrability for the defect system with a defect at a fixed location. Here we show that analogous discussions can be adapted to the case of the timedependent defect systems.

To fix ideas, we concentrate on the NLS equation. Let us first recall some important results regarding the multi-symplectic formalism of the NLS equation [14]. The key observation in [14] is to introduce the following new equal-space Poisson bracket

$$
\left\{u(x, t), u_{x}^{*}(x, \tau)\right\}=-\delta(t-\tau), \quad\left\{u_{x}(x, t), u^{*}(x, \tau)\right\}=\delta(t-\tau)
$$

and

$$
\{u(x, t), u(x, \tau)\}=\left\{u(x, t), u^{*}(x, \tau)\right\}=\left\{u_{x}(x, t), u(x, \tau)\right\}=\left\{u_{x}(x, t), u_{x}(x, \tau)\right\}=0 .
$$

With this Poisson bracket, the NLS equation (2.3) can be written in the following Hamiltonian form

$$
u_{x x}=\left\{u_{x}, H_{T}\right\},
$$

where the new Hamiltonian $H_{T}$ is given by

$$
H_{T}=\int_{-\infty}^{\infty} \mathrm{d} \tau\left(-\left|u_{x}\right|^{2}-\frac{\mathrm{i}}{2}\left(u^{*} u_{\tau}-u_{\tau}^{*} u\right)+\varepsilon|u|^{4}\right)
$$

We construct a transition matrix from the time-part of the Lax pair: Transition matrix $M_{T}(x, t, \lambda)$ is defined as the fundamental solution of the auxiliary linear problem $(2.1 b)$ with $M_{T}(x,-\infty, \lambda)=I$ (here I denotes identity matrix),

$$
M_{T}(x, t, \lambda)=\underset{\exp }{\curvearrowleft} \int_{-\infty}^{t} V(x, \tau, \lambda) \mathrm{d} \tau .
$$

Using the Poisson bracket (4.12), one can check directly that $M_{T}(x, t, \lambda)$ satisfies the following $r$-matrix relation [14]:

$$
\left\{M_{T 1}(x, t, \lambda), M_{T 2}(x, t, \mu)\right\}=\left[r(\lambda-\mu), M_{T}(x, t, \lambda) \otimes M_{T}(x, t, \mu)\right],
$$

where $M_{T 1}(x, t, \lambda)=M_{T}(x, t, \lambda) \otimes I, M_{T 2}(x, t, \mu)=I \otimes M_{T}(x, t, \mu)$ and

$$
r(\lambda-\mu)=\frac{\varepsilon}{2(\lambda-\mu)}\left(\begin{array}{llll}
1 & 0 & 0 & 0 \\
0 & 0 & 1 & 0 \\
0 & 1 & 0 & 0 \\
0 & 0 & 0 & 1
\end{array}\right) .
$$

As an application, we can deduce that integrals of motion constructed from the trace of the monodromy matrix $M_{T}(x, \infty, \lambda)$ are in involution with respect to the Poisson bracket (4.12). Thus, Liouville integrability of the NLS equation with respect to the Poisson bracket (4.12) is proved. For a system without a defect, the above argument for Liouville integrability is equivalent to the standard argument with respect to the usual (equal-time) Poisson bracket (see [28] for details). The advantage of the above argument is that it can be applied to establish Liouville integrability of a system with a defect; see [14] for the case of the NLS equation with a defect being fixed at $x=x_{0}$ and see the following discussions for the system with time-dependent defect. 
We now adapt the arguments of [24,25] about canonical transformations to the above new Poisson bracket: Transformation, which maps $u$ to $\tilde{u}$, is canonical if the following Pfaffian form is relative integrable invariant

$$
\int_{-\infty}^{\infty} \mathrm{d} t\left(u_{x}^{*} \mathrm{~d} u+u_{x} \mathrm{~d} u^{*}\right)+H_{T} \mathrm{~d} x
$$

That is

$$
\int_{-\infty}^{\infty} \mathrm{d} t\left(\tilde{u}_{x}^{*} \mathrm{~d} \tilde{u}+\tilde{u}_{x} \mathrm{~d} \tilde{u}^{*}\right)+\tilde{H}_{T} \mathrm{~d} x=\int_{-\infty}^{\infty} \mathrm{d} t\left(u_{x}^{*} \mathrm{~d} u+u_{x} \mathrm{~d} u^{*}\right)+H_{T} \mathrm{~d} x-\mathrm{d} W
$$

Here

$$
W\left(u, u^{*}, \tilde{u}, \tilde{u}^{*} ; x\right)=F\left(u, u^{*}, \tilde{u}, \tilde{u}^{*}\right)-E x
$$

(with $E$ being a real constant) is called a generator of the transformation. From (4.19), we obtain the transformation equations:

and

$$
\left.\begin{array}{l}
u_{x}=\frac{\delta F}{\delta u^{*}}, \quad u_{x}^{*}=\frac{\delta F}{\delta u} \\
\tilde{u}_{x}=-\frac{\delta F}{\delta \tilde{u}^{*}}, \quad \tilde{u}_{x}^{*}=-\frac{\delta F}{\delta \tilde{u}} .
\end{array}\right\}
$$

For the NLS equation, we find that $F$ can be taken as

$$
F=\int_{-\infty}^{\infty} \mathrm{d} t\left(\frac{\mathrm{i}}{2} \varepsilon \Omega\left(\frac{\tilde{u}_{t}-u_{t}}{\tilde{u}-u}-\frac{\tilde{u}_{t}^{*}-u_{t}^{*}}{\tilde{u}^{*}-u^{*}}\right)+\frac{1}{3} \varepsilon \Omega^{3}-\Omega\left(|u|^{2}+|\tilde{u}|^{2}+\varepsilon \alpha^{2}\right)+\mathrm{i} \alpha\left(u \tilde{u}^{*}-u^{*} \tilde{u}\right)\right) .
$$

Then, the transformation equation (4.21) becomes nothing but the BT (2.13) of the NLS equation. Hence, the defect condition for the NLS equation can be interpreted as a canonical transformation with respect to the Poisson bracket (4.12).

We now turn to the implementation of the classical $r$-matrix approach to the NLS equation in the presence of the time-dependent defect. For the time-dependent defect NLS system, we define the transition matrix as follows

$$
\mathcal{M}(x, t, \lambda)= \begin{cases}\tilde{M}_{T}(x, t, \lambda), & -\infty<x<c(t), \\ M_{T}(x, t, \lambda), & c(t) \leq x<\infty,\end{cases}
$$

where $\tilde{M}_{T}(x, t, \lambda)$ is the analogous matrix of $M_{T}(x, t, \lambda)$ but defined by the new canonical variable $\tilde{u}$. Due to the canonical property of the transformation, we immediately conclude that $\mathcal{M}(x, t, \lambda)$ satisfies the same $r$-matrix relation as that of $M_{T}(x, t, \lambda)$, that is

$$
\left\{\mathcal{M}_{1}(x, t, \lambda), \mathcal{M}_{2}(x, t, \mu)\right\}=[r(\lambda-\mu), \mathcal{M}(x, t, \lambda) \otimes \mathcal{M}(x, t, \mu)] .
$$

As a result, the trace of the monodromy matrix $\mathcal{M}(x, \infty, \lambda)$ provides a generating function for the conserved quantities that are in involution with respect to the Poisson bracket (4.12). Thus, we establish Liouville integrability of the time-dependent defect NLS system (3.1).

\section{The case of multiple time-dependent defects}

In this section, we generalize the above arguments for integrability to the case that there are multiple time-dependent defects in a system.

Let us first fix some notations. We assume that $c_{1}(t), c_{2}(t), \ldots, c_{n}(t)$ are $n$ functions of class $C^{1}$ such that $c_{1}(t)<c_{2}(t)<\cdots<c_{n}(t)$. We consider $n+1$ auxiliary problems for $\phi^{(j)}, j=0, \ldots, n$, with Lax pair $U^{(j)}, V^{(j)}$ defined as in (2.1) with the fields $u^{(j)}, v^{(j)}$, replacing $u, v$. We assume that the auxiliary problem for $U^{(0)}, V^{(0)}$ exists for $x<c_{1}(t)$, the one for $U^{(j)}, V^{(j)}$ exists for $c_{j}(t)<x<c_{j+1}(t)$, $j=1, \ldots, n-1$, and the one for $U^{(n)}, V^{(n)}$ exists for $x>c_{n}(t)$. At $x=c_{j}(t), j=1, \ldots, n$, we assume 
that the two systems are connected via the condition

$$
\phi^{(j-1)}\left(c_{j}(t), t, \lambda\right)=B^{(j)}\left(c_{j}(t), t, \lambda\right) \phi^{(j)}\left(c_{j}(t), t, \lambda\right),
$$

where $B^{(j)}(x, t, \lambda), j=1, \ldots, n$, satisfy

$$
B_{x}^{(j)}(x, t, \lambda)=U^{(j-1)}(x, t, \lambda) B^{(j)}(x, t, \lambda)-B^{(j)}(x, t, \lambda) U^{(j)}(x, t, \lambda)
$$

and

$$
B_{t}^{(j)}(x, t, \lambda)=V^{(j-1)}(x, t, \lambda) B^{(j)}(x, t, \lambda)-B^{(j)}(x, t, \lambda) V^{(j)}(x, t, \lambda) .
$$

Given these notations, we find the following proposition, whose proof is similar to that of proposition 4.1 .

Proposition 5.1. In the presence of multiple time-dependent defects, the generating function for the integrals of motion reads

$$
I(\lambda)=\int_{-\infty}^{c_{1}(t)} u^{(0)} \Gamma^{(0)} \mathrm{d} x+\sum_{j=1}^{n-1} \int_{c_{j}(t)}^{c_{j+1}(t)} u^{(j)} \Gamma^{(j)} \mathrm{d} x+\int_{c_{n}(t)}^{\infty} u^{(n)} \Gamma^{(n)} \mathrm{d} x+I_{\text {defect }}(\lambda),
$$

where

$$
I_{\text {defect }}(\lambda)=-\left.\sum_{j=1}^{n} \ln \left(B_{11}^{(j)}+B_{12}^{(j)} \Gamma^{(j)}\right)\right|_{x=c_{j}(t)}
$$

Here $B_{11}^{(j)}$ and $B_{12}^{(j)}$ denote, respectively, the 11-entry and 12-entry of the defect matrix $B^{(j)}$.

The classical $r$-matrix approach can also be implemented in the system with multiple defects. Indeed, we define the transition matrix for the defect NLS equation as follows

$$
\mathcal{M}(x, t, \lambda)= \begin{cases}M_{T}^{(0)}(x, t, \lambda), & -\infty<x<c_{1}(t), \\ M_{T}^{(j)}(x, t, \lambda), & c_{j}(t) \leq x<c_{j+1}(t), \quad j=1, \ldots, n-1, \\ M_{T}^{(n)}(x, t, \lambda), & c_{n}(t) \leq x<\infty\end{cases}
$$

where

$$
M_{T}^{(j)}(x, t, \lambda)=\exp \int_{-\infty}^{t} V^{(j)}(x, \tau, \lambda) \mathrm{d} \tau, \quad j=0,1, \ldots, n .
$$

Then $\mathcal{M}(x, t, \lambda)$ satisfies the same $r$-matrix relation as that of (4.23). This fact immediately yields the Poisson commutativity of the motion integrals that generated from the trace of the monodromy matrix $\mathcal{M}(x, \infty, \lambda)$.

\section{Soliton solutions meeting the defects}

It is now our aim to seek soliton solutions that can meet the defect of a system. To fix our ideas, we consider the KdV equation as an illustrative example. We will focus on an interesting case: the defect moves at a constant speed. We will show that the KdV equation with such a timedependent defect admits peakon solutions.

Recall that a single-soliton for the $\mathrm{KdV}$ equation is given by

$$
u(x, t)=\frac{8 k^{2}}{\left(\alpha_{1} \exp (\xi)+\alpha_{1}^{-1} \exp (-\xi)\right)^{2}}, \quad \xi=k\left(x-4 k^{2} t\right), k>0,
$$


where $\alpha_{1}$ is a positive constant. We assume that the defect takes place at $x=4 k^{2} t$ (i.e. the speed of the defect is in coincidence with the wave speed). In the presence of such a defect, we take the soliton on the other side of the defect in a similar form

$$
\tilde{u}(x, t)=\frac{8 k^{2}}{\left(\alpha_{2} \exp (\xi)+\alpha_{2}^{-1} \exp (-\xi)\right)^{2}}, \quad \xi=k\left(x-4 k^{2} t\right), k>0,
$$

where $\alpha_{2}$ is a parameter to be determined by the defect condition. By applying the defect condition (3.2c) and (3.2d), we find that $\alpha_{2}$ is determined by

$$
\begin{aligned}
2 k\left(\frac{\alpha_{1}-\alpha_{1}^{-1}}{\left(\alpha_{1}+\alpha_{1}^{-1}\right)^{3}}+\frac{\alpha_{2}-\alpha_{2}^{-1}}{\left(\alpha_{2}+\alpha_{2}^{-1}\right)^{3}}\right)= & \left(\frac{1}{\left(\alpha_{1}+\alpha_{1}^{-1}\right)^{2}}-\frac{1}{\left(\alpha_{2}+\alpha_{2}^{-1}\right)^{2}}\right) \\
& \times \sqrt{\beta^{2}-16 k^{2}\left(\frac{1}{\left(\alpha_{1}+\alpha_{1}^{-1}\right)^{2}}+\frac{1}{\left(\alpha_{2}+\alpha_{2}^{-1}\right)^{2}}\right) .} .
\end{aligned}
$$

We further restrict our attention to find a solution such that there is no discontinuity at the defect. This requirement implies that

$$
\left(\alpha_{1}+\alpha_{1}^{-1}\right)^{2}=\left(\alpha_{2}+\alpha_{2}^{-1}\right)^{2} .
$$

A non-trivial solution for $\alpha_{2}$ satisfying both (6.3) and (6.4) is that $\alpha_{2}=\alpha_{1}^{-1}$. Let $\alpha_{1}=\exp \gamma$. The solutions (6.1) and (6.2) on each side of the defect can be written in a uniform form: $u(x, t)=$ $2 k^{2} \operatorname{sech}^{2}(|\xi|+\gamma)$. To sum up, we find

Proposition 6.1. Let the defect move at a constant speed $x=4 k^{2} t$, and let the defect condition be defined by (3.2c) and (3.2d) with $x=c(t)$ replaced by $x=4 k^{2} t$. The KdV equation with such a defect admits the following single-peakon solution

$$
u(x, t)=2 k^{2} \operatorname{sech}^{2}(|\xi|+\gamma), \quad \xi=k\left(x-4 k^{2} t\right) .
$$

If $\gamma>0,(6.5)$ presents a peakon wave with discontinuous first derivative at the peak (at $\xi=0)$; see figure 1 for a profile of this wave. If $\gamma<0,(6.5)$ presents a wave with two peaks (at $\xi= \pm \gamma$ ) pointing upwards and one peak (at $\xi=0$ ) pointing downwards (called an anti-peakon simply), where the first derivative is discontinuous at $\xi=0$ (the position of the anti-peakon); see figure 2 for a profile of this wave. We note that the existence of peakon solutions was known as a typical feature of the $\mathrm{CH}$ type equations [20,21]. Here our results show that the usual soliton equations in the presence of time-dependent defects (such as the defect $\mathrm{KdV}$ equation discussed above) can also admit peakon solutions. We should emphasize that peakon solutions for the $\mathrm{CH}$ type equations and peakon solutions presented here should be interpreted in two different senses: the former ones should be interpreted in a suitable weak sense, while the latter ones should be interpreted in the sense that there is a time-dependent defect.

We now extend the above results to the case of the $\mathrm{KdV}$ equation with multiple defects located at different positions. For clarity, we will assume, in the following, $\gamma>0$ and the defects located, respectively, at $\xi=j \gamma, j=0, \pm 1, \ldots, \pm N$. As above, we restrict our attention to a solution which is continuous at the defects. In analogy with the analysis used above, we find

Proposition 6.2. Assume that $\gamma>0$ and the defects locate at $\xi=j \gamma, j=0, \pm 1, \ldots, \pm N$. Let the defect conditions be defined by (3.2c) and (3.2d) with $x=c(t)$ replaced by $x=4 k^{2} t+j k^{-1} \gamma, j=0, \pm 1, \ldots, \pm N$. The KdV equation with such multiple defects admits the following multi-peakon solution

$$
u= \begin{cases}2 k^{2} \operatorname{sech}^{2}(|\xi+N \gamma|+\gamma), & -\infty<\xi \leq-(N-1) \gamma, \\ 2 k^{2} \operatorname{sech}^{2}(|\xi+(N-2 j) \gamma|+\gamma), & (2 j-1-N) \gamma<\xi \leq(2 j+1-N) \gamma, 1 \leq j \leq N, \\ 2 k^{2} \operatorname{sech}^{2}(|\xi-N \gamma|+\gamma), & (N+1) \gamma<\xi<\infty,\end{cases}
$$

where $\xi=k\left(x-4 k^{2} t\right)$.

The above solution (6.6) represents a wave which has $(N+1)$ peakons at $\xi=(2 m-N) \gamma, m=$ $0,1, \ldots N$, and $N$ anti-peakons at $\xi=(2 m-1-N) \gamma, m=1, \ldots N$. For example, for $N=1$, it has 


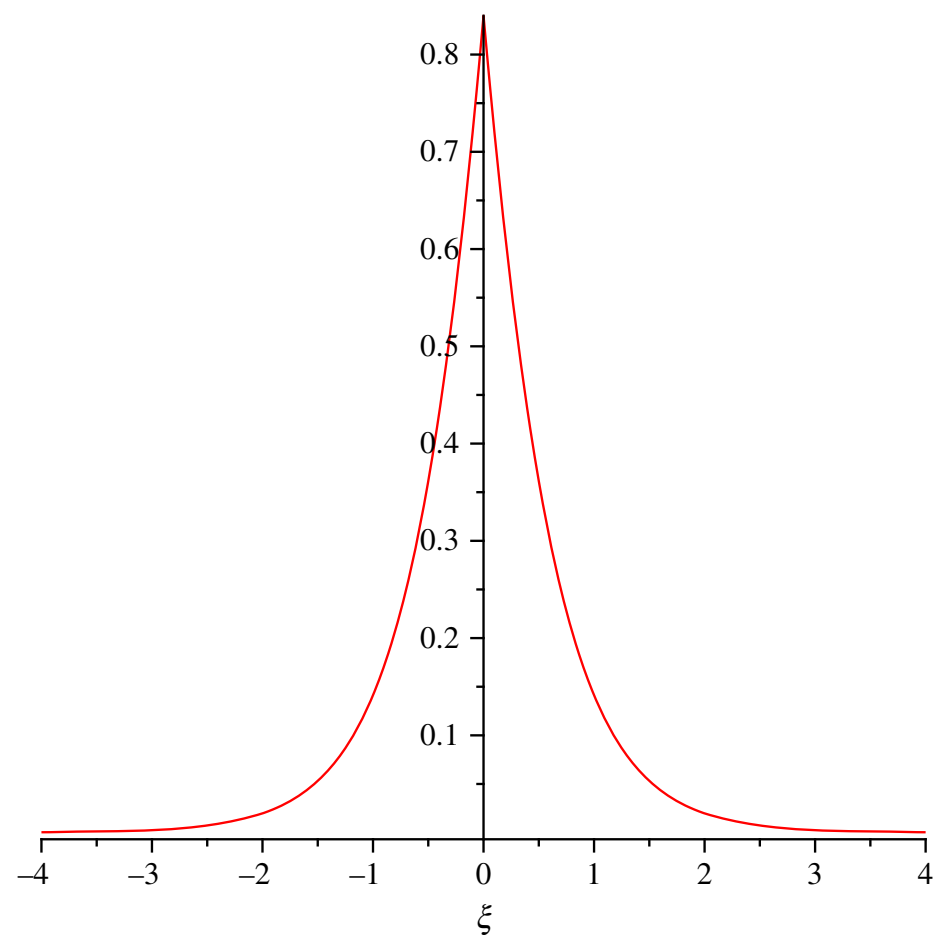

Figure 1. The single-peakon solution determined by (6.5) with parameters $k=\gamma=1$. (Online version in colour.)

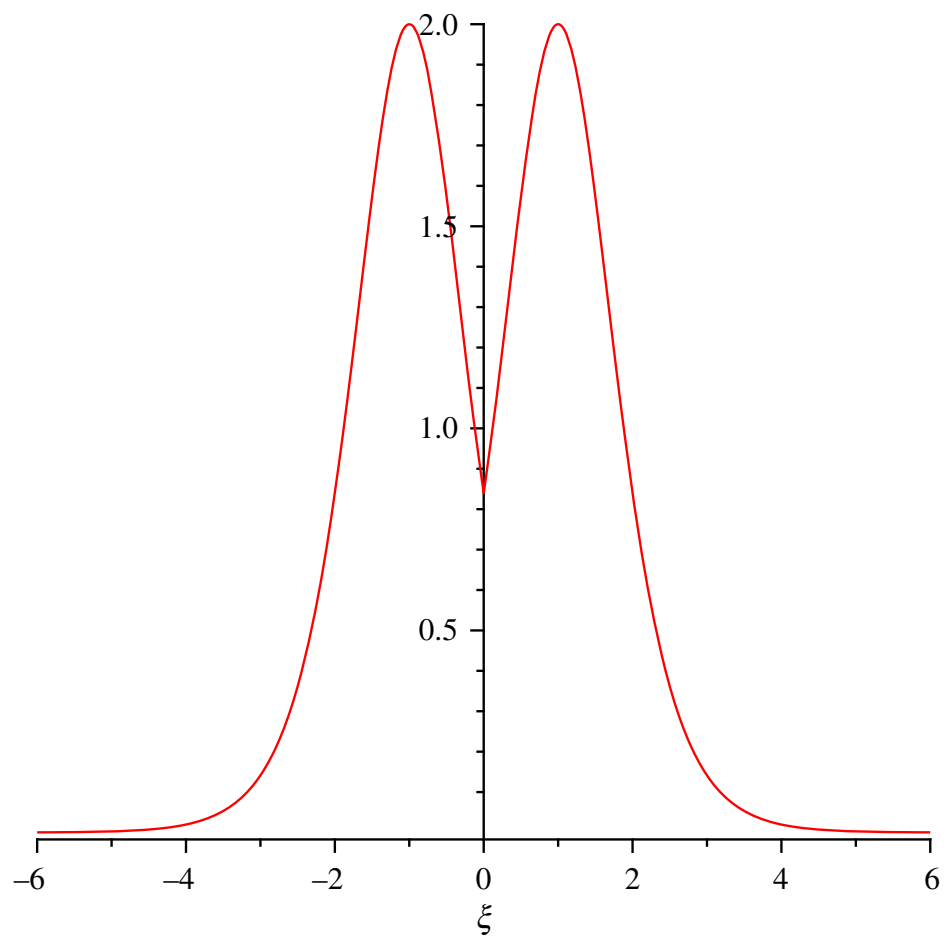

Figure 2. The solution determined by (6.5) with parameters $k=-\gamma=1$. (Online version in colour.) 


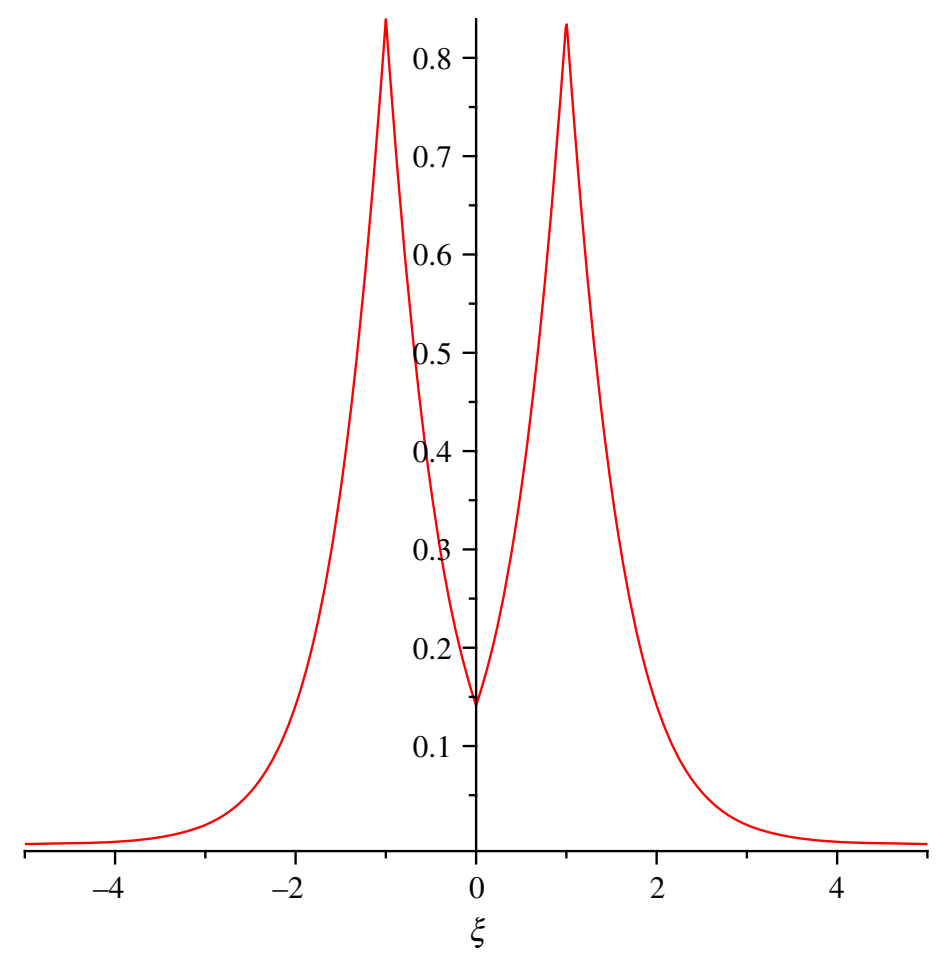

Figure 3. The M-shape peakon solution determined by (6.6) with $N=1$ and parameters $k=\gamma=1$. (Online version in colour.)

two peakons at $\xi= \pm \gamma$, and one anti-peakon at $\xi=0$, and it looks like a ' $\mathrm{M}$ ' shape wave; see figure 3 for a profile of this M-shape wave solution.

Remark 6.3. The above solutions are derived by a direct ansatz for the fields to either side of the defect tuned to satisfy the defect condition. The fact that the defect condition is corresponding to a BT implies that we can systematically construct the solution of the defect system in the following way. Given a solution $u(x, t)$ of the bulk system for $x \in(c(t), \infty)$, we first implement a BT for all $x, t$ to find $\tilde{u}(x, t)$. Then we define $\tilde{u}(x, t)$ as the solution of the bulk system for $x \in(-\infty, c(t))$. The solution constructed in such a manner solves the equation in the bulk as well as satisfies the defect condition at $x=c(t)$, thus it provides a solution of the defect system. For the case of the defect being fixed, this strategy has been employed recently in [18] to construct finite-gap solutions for the defect $\mathrm{KdV}$ and sine-Gordon equations. For the case of the defect moving with time as presented in this paper, similar considerations will be investigated in the future.

\section{Concluding remarks}

We have studied $(1+1)$-dimensional integrable soliton equations associated with the AKNS system in the presence of time-dependent defects. We defined the defect condition as a Bäcklund transformation evaluated at the time-dependent defect location. We demonstrated that the resulting defect systems admit Lagrangian descriptions and established the integrability of the resulting defect systems both by constructing an infinite set of conserved densities and by implementing the classical $r$-matrix method. We also studied soliton solutions for the defect systems. Although our results are presented for integrable soliton equations in the continuous case, it is clear that analogous results can be applied to integrable soliton equations in the discrete case, such as the integrable discrete NLS equation and the Toda lattice equation. 
Data accessibility. This article has no additional data.

Authors' contributions. B.X. conceived the research idea. R.Z. discussed the results. All authors participated in

drafting the manuscript.

Competing interests. We declare we have no competing interests.

Funding. This work was supported by the National Natural Science Foundation of China (grant nos 11771186 and 11671177).

Acknowledgements. The authors would like to express their sincerest thanks to the referees for their valuable comments, which have helped us to improve this paper.

\section{References}

1. Bibikov PN, Tarasov VO. 1989 A boundary-value problem for the nonlinear Schrödinger equation. Theor. Math. Phys. 79, 334-579. (doi:10.1007/BF01016541)

2. Goodman RH, Holmes PJ, Weinstein MI. 2002 Interaction of sine-Gordon kinks with defects: phase space transport in a two-mode model. Physica D 161, 21-44. (doi:10.1016/S0167-2789(01)00353-0)

3. Cao XD, Malomed BA. 1995 Soliton-defect collisions in the nonlinear Schrödinger equation. Phys. Lett. A 206, 177-182. (doi:10.1016/0375-9601(95)00611-6)

4. Holmer J, Marzuola J, Zworski M. 2007 Fast soliton scattering by delta impurities. Commun. Math. Phys. 274, 187-216. (doi:10.1007/s00220-007-0261-z)

5. Bowcock P, Corrigan E, Zambon C. 2004 Classically integrable field theories with defects. Int. J. Mod. Phys. A 19, 82-91 (doi:10.1142/S0217751X04020324)

6. Bowcock P, Corrigan E, Zambon C. 2004 Affine Toda field theories with defects. J. High Energy Phys. 2004, 056. (doi:10.1088/1126-6708/2004/01/056)

7. Bowcock P, Corrigan E, Zambon C. 2005 Some aspects of jump-defects in the quantum sineGordon model. J. High Energy Phys. 508, 23. (doi:10.1088/1126-6708/2005/08/023)

8. Corrigan E, Zambon C. 2004 Aspects of sine-Gordon solitons, defects and gates. J. Phys. A 37, L471-L477. (doi:10.1088/0305-4470/37/37/L03)

9. Corrigan E, Zambon C. 2006 Jump-defects in the nonlinear Schrödinger model and other nonrelativistic field theories. Nonlinearity 19, 1447-1469. (doi:10.1088/0951-7715/19/6/012)

10. Corrigan E, Zambon C. 2009 A new class of integrable defects. J. Phys. A 42, 475203. (doi:10.1088/1751-8113/42/47/475203)

11. Zambon C. 2014 The classical nonlinear Schrödinger model with a new integrable boundary. J. High Energy Phys. 2014, 36. (doi:10.1007/JHEP08(2014)036)

12. Habibullin I, Kundu A. 2008 Quantum and classical integrable sine-Gordon model with defect. Nucl. Phys. B 795, 549-568. (doi:10.1016/j.nuclphysb.2007.11.022)

13. Caudrelier V. 2008 On a systematic approach to defects in classical integrable field theories. Int. J. Geom. Meth. Mod. Phys. 5, 1085-1108. (doi:10.1142/S0219887808003223)

14. Caudrelier V, Kundu A. 2015 A multisymplectic approach to defects in integrable classical field theory. J. High Energy Phys. 2, 88. (doi:10.1007/JHEP02(2015)088)

15. Avan J, Doikou A. 2012 Liouville integrable defects: the non-linear Schrödinger paradigm. J. High Energy Phys. 1, 40. (doi:10.1007/JHEP01(2012)040)

16. Avan J, Doikou A. 2012 The sine-Gordon model with integrable defects revisited. J. High Energy Phys. 2012, 8. (doi:10.1007/JHEP11(2012)008)

17. Doikou A. 2016 Classical integrable defects as quasi Bäcklund transformations. Nucl. Phys. B 911, 212-230. (doi:10.1016/j.nuclphysb.2016.08.006)

18. Corrigan E, Parini R. 2017 Type I integrable defects and finite-gap solutions for KdV and sine-Gordon models. J. Phys. A 50, 284001. (doi:10.1088/1751-8121/aa7612)

19. Ablowitz MJ, Kaup DJ, Newell AC, Segur H. 1974 The inverse scattering transform-Fourier analysis for nonlinear problems. Stud. Appl. Math. 53, 249-315. (doi:10.1002/sapm1974534249)

20. Camassa R, Holm DD. 1993 An integrable shallow water equation with peaked solitons. Phys. Rev. Lett. 71, 1661-1664. (doi:10.1103/PhysRevLett.71.1661)

21. Fuchssteiner B, Fokas AS. 1981 Symplectic structures, their Bäcklund transformation and hereditary symmetries. Physica D 4, 47-66. (doi:10.1016/0167-2789(81)90004-X)

22. Matveev VB, Salle MA. 1991 Darboux transformations and solitons. Berlin, Germany: SpringerVerlag.

23. Rogers C, Schief WK. 2002 Bäcklund and Darboux transformations: geometry and modern applications in soliton theory. Cambridge, UK: Cambridge University Press. 
24. Kodama Y, Wadati M. 1976 Theory of canonical transformations for nonlinear evolution equations. I. Prog. Theor. Phys. 56, 1740-1755. (doi:10.1143/PTP.56.1740)

25. Kodama Y. 1977 Theory of canonical transformations for nonlinear evolution equations. II. Prog. Theor. Phys. 57, 1900-1916. (doi:10.1143/PTP.57.1900)

26. Sklyanin EK. 1979 On complete integrability of the Landau-Lifshitz equation. Preprint Leningrad Branch of the Mathematics Institute, E-3-79.

27. Sklyanin EK. 1988 Boundary conditions for integrable quantum systems. J. Phys. A 21, 23752389. (doi:10.1088/0305-4470/21/10/015)

28. Faddeev LD, Takhtajan LA. 2007 Hamiltonian methods in the theory of solitons. Berlin, Germany: Springer. 